\title{
BMJ Open Rapid Emergency Medicine Score (REMS) in the trauma population: a retrospective study
}

\author{
Bryan F Imhoff, ${ }^{1}$ Nia J Thompson, ${ }^{1}$ Michael A Hastings, ${ }^{2}$ Niaman Nazir, ${ }^{3}$ \\ Michael Moncure, ${ }^{4}$ Chad M Cannon ${ }^{5}$
}

To cite: Imhoff BF,

Thompson NJ, Hastings MA, et al. Rapid Emergency Medicine Score (REMS) in the trauma population: a retrospective study. BMJ Open 2014;4: 0004738 . doi:10.1136/bmjopen-2013004738

- Prepublication history for this paper is available online. To view these files please visit the journal online (http://dx.doi.org/10.1136/ bmjopen-2013-004738).

Received 20 December 2013 Revised 31 March 2014 Accepted 11 April 2014

\section{(a) CrossMark}

${ }^{1}$ The University of Kansas School of Medicine, Kansas City, Kansas, USA

${ }^{2}$ Department of Trauma Services, Dell Children's Medical Central of Central Texas, Austin, Texas, USA ${ }^{3}$ Department of Preventive Medicine and Public Health, The University of Kansas Hospital, Kansas City, Kansas, USA

${ }^{4}$ Department of Surgery, The University of Kansas Hospital, Kansas City, Kansas, USA

${ }^{5}$ Department of Emergency Medicine, University of

Kansas Hospital, Kansas City, Kansas, USA

\section{Correspondence to} Dr Chad M Cannon; ccannon@kumc.edu

\section{ABSTRACT}

Objective: Rapid Emergency Medicine Score (REMS) is an attenuated version of the Acute Physiology and Chronic Health Evaluation (APACHE) II score and has utility in predicting mortality in non-surgical patients, but has yet to be tested among the trauma population. The objective was to evaluate REMS as a risk stratification tool for predicting in-hospital mortality in traumatically injured patients and to compare REMS accuracy in predicting mortality to existing trauma scores, including the Revised Trauma Score (RTS), Injury Severity Score (ISS) and Shock Index (SI). Design and setting: Retrospective chart review of the trauma registry from an urban academic American College of Surgeons (ACS) level 1 trauma centre.

Participants: 3680 patients with trauma aged 14 years and older admitted to the hospital over a 4year period. Patients transferred from other hospitals were excluded from the study as were those who suffered from burn or drowning-related injuries. Patients with vital sign documentation insufficient to calculate an REMS score were also excluded.

Primary outcome measures: The predictive ability of REMS was evaluated using ORs for in-hospital mortality. The discriminate power of REMS, RTS, ISS and SI was compared using the area under the receiver operating characteristic curve.

Results: Higher REMS was associated with increased mortality $(p<0.0001)$. An increase of 1 point in the 26point REMS scale was associated with an OR of 1.51 for in-hospital death ( $95 \% \mathrm{Cl} 1.45$ to 1.58). REMS (area under the curve (AUC) $0.91 \pm 0.02$ ) was found to be similar to RTS (AUC $0.89 \pm 0.04$ ) and superior to ISS (AUC $0.87 \pm 0.01$ ) and SI (AUC 0.55 \pm 0.31 ) in predicting in-hospital mortality.

Conclusions: In the trauma population, REMS appears to be a simple, accurate predictor of inhospital mortality. While REMS performed similarly to RTS in predicting mortality, it did outperform other traditionally used trauma scoring systems, specifically ISS and SI.

\section{INTRODUCTION}

Trauma and unintentional injury are the leading causes of death for all individuals less

\section{Strengths and limitations of this study}

- This is the first study to demonstrate the applicability of Rapid Emergency Medicine Score (REMS), a more rapid and less invasive version of the Acute Physiology and Chronic Health Evaluation (APACHE) II score, to traumatically injured patients.

- REMS, which is calculated from readily available parameters, performs favourably in comparison to existing trauma scores to predict in-hospital mortality.

- While the study analysed a large sample size at a single urban academic trauma centre, attempts to extrapolate results to other trauma populations may not be reliable.

- REMS was originally derived from the medicine population, therefore the scoring system does not differentiate between injury types, which are known factors in predicting mortality.

- The expertise of the treating trauma centre will influence patient outcomes and therefore will impact the REMS-mortality relationship. This however is a limitation of all scoring systems and is not unique to REMS alone.

than 44 years of age which result in a major cost burden for the healthcare system. ${ }^{1}$ Current literature supports that early diagnosis and appropriate treatment both improve outcomes and are cost-effective. Over the past decade, scoring systems have been utilised to assess injury severity and provide an objective measure for treatment and appropriate allocation of healthcare resources. The Acute Physiology and Chronic Health Evaluation (APACHE II) is a validated scale that assesses severity of illness among non-surgical, surgical and intensive care hospital patients. ${ }^{2}$ The score incorporates body temperature, respiratory rate (RR), heart rate, mean arterial pressure (MAP), oxygenation of arterial blood, arterial $\mathrm{pH}$, serum sodium and potassium levels, serum creatinine, haematocrit, white cell count and Glasgow Coma Scale (GCS). ${ }^{3}$ 
With a reliance on laboratory tests such as blood chemistry analysis, APACHE II scoring remains impractical for rapid injury severity assessment required in the emergency department (ED) or in the field. The Rapid Emergency Medicine Score (REMS), an attenuated version of APACHE II, allows for prompt calculation. ${ }^{2} 4$ REMS is a composite score consisting of the GCS, RR, oxygen saturation, MAP, hazard ratio and age. ${ }^{5}$ Among non-surgical patients who present to the ED, REMS has proven to be a valid predictor of mortality.

While many ED scoring methodologies focus on evaluating short-term outcomes, REMS has been shown to predict mortality at 1 week (hazard ratio $1.34,95 \%$ CI 1.30 to 1.37 ), 1 month (hazard ratio $1.30,95 \%$ CI 1.27 to 1.32 ) and 3 months (hazard ratio $1.26,95 \%$ CI 1.24 to 1.28$).{ }^{2}$ An additional study comparing area under the receiver operating curve (AUC) for the Rapid Acute Physiology Score $(0.64,95 \%$ CI 0.59 to 0.69$)$ and REMS $(0.74,95 \%$ CI 0.70 to 0.78$)$ in non-surgical ED patients determined superiority of the REMS. ${ }^{5}{ }^{6}$ Every point increase in the 26-point REMS score was associated with an OR of $1.40(95 \%$ CI 1.36 to $1.45, \mathrm{p}<0.0001)$ for in-hospital mortality. ${ }^{5}$ While there have been extensive publications reporting sound predicative validity for this score among non-surgical patients, the utility of REMS in the trauma population has yet to be analysed.

The purpose of this study was to evaluate REMS as a risk stratification tool to predict in-hospital mortality in traumatically injured patients. Secondary objectives included comparing REMS to the Revised Trauma Score (RTS), the Injury Severity Score (ISS) and the Shock Index (SI) to determine which scoring system was a superior predictor of mortality, in addition to examining the six components of REMS to determine which, if any, were most predictive of mortality.

\section{MATERIALS AND METHODS}

\section{Study design}

This was a retrospective chart review of the trauma registry at an urban academic American College of Surgeons (ACS) level 1 trauma centre. The study was reviewed by the hospital institutional review board, The University of Kansas Medical Center Human Subjects Committee and a waiver of informed consent was granted.

\section{Study population}

Analysis focused on 3680 patients with trauma aged 14 years and older admitted to the hospital over a 4-year period. Patients transferred from other hospitals were excluded from the study; for these patients, vital signs may have changed from initial values due to treatment at institution, affecting REMS calculations. Patients who suffered from burn or drowning-related injuries were also excluded from the study. Finally, 158 patients with vital sign documentation that was insufficient for REMS calculation were also excluded. Non-REMS injury score data (ie, SI, ISS, RTS) were missing from 47 patients; these patients were included in all the REMS analyses except for calculation of the AUC for their respective scoring systems.

\section{Protocol}

Variables collected included age, date and time of arrival, race, sex, systolic blood pressure (SBP), diastolic blood pressure, RR, heart rate, oxygen saturation, length of stay and GCS. The ISS and RTS scores were calculated by and obtained from the trauma registry. The MAP and SI (heart rate divided by SBP) were calculated during data analysis.

\section{Measurements}

REMS scoring requires RR, heart rate, MAP, GCS, age and oxygen saturation. ${ }^{5}$ In REMS calculation, age is assigned a value from 0 to 6 , and the remaining five variables are each assigned a score from 0 to 4 (table 1). REMS is a sum of these values with a maximum composite score of 26 , with higher values being indicative of worse prognosis.

In this study, REMS was compared to three currently utilised injury scoring systems, including SI, ISS and RTS, to determine which measure was superior in predicting mortality. SI is calculated by dividing heart rate by SBP and has proven useful in predicting mortality and the severity of illness in traumatically ill patients. ${ }^{78}$ Likewise, the ISS correlates with mortality. In contrast,

\begin{tabular}{|c|c|c|c|c|c|c|c|}
\hline \multirow[b]{2}{*}{ Variable } & \multicolumn{7}{|l|}{ Score } \\
\hline & 0 & +1 & +2 & +3 & +4 & +5 & +6 \\
\hline Age (years) & $<45$ & & $45-54$ & $55-64$ & & $65-74$ & $>74$ \\
\hline \multirow[t]{2}{*}{$\operatorname{MAP}(\mathrm{mm} \mathrm{Hg})$} & 70-109 & & $110-129$ & $130-159$ & $>159$ & & \\
\hline & & & $50-69$ & & $\leq 49$ & & \\
\hline \multirow[t]{2}{*}{ Heart rate $(\mathrm{bpm})$} & 70-109 & & 110-139 & $140-179$ & $>179$ & & \\
\hline & & & $55-69$ & $40-54$ & $\leq 39$ & & \\
\hline \multirow[t]{2}{*}{ RR (breaths/min) } & $12-24$ & $25-34$ & $6-9$ & $35-49$ & $>49$ & & \\
\hline & & $10-11$ & & & $\leq 5$ & & \\
\hline $\mathrm{O}_{2}$ saturation (\%) & $>89$ & $86-89$ & & $75-85$ & $<75$ & & \\
\hline GCS & 14 or 15 & $11-13$ & $8-10$ & $5-7$ & 3 or 4 & & \\
\hline
\end{tabular}


Table 2 Baseline characteristics and hospital course for 3680 patients with trauma

\begin{tabular}{|c|c|c|c|}
\hline & $\begin{array}{l}\text { Dead }(\mathrm{N}=191) \\
\text { Mean }(\mathrm{SD})^{\star}\end{array}$ & $\begin{array}{l}\text { Alive }(\mathrm{N}=3489) \\
\text { Mean }(\mathrm{SD})^{\star}\end{array}$ & p Value \\
\hline Age (years) & $43.7(21.0)$ & $36.5(17.0)$ & $<0.0001$ \\
\hline Male (\%) & 77.0 & 73.7 & 0.0472 \\
\hline Race (\%) & & & 0.0564 \\
\hline White & 59.2 & 62.4 & \\
\hline Black & 23.5 & 23.2 & \\
\hline Other & 17.3 & 14.4 & \\
\hline Length of stay (days) & $4.4(8.0)$ & $7.6(15.2)$ & 0.0043 \\
\hline Systolic BP (mm Hg) & $104.1(68.3)$ & $142.5(24.9)$ & $<0.0001$ \\
\hline Diastolic BP (mm Hg) & $61.1(43.6)$ & $84.6(20.5)$ & $<0.0001$ \\
\hline Heart rate $(\mathrm{bpm})$ & $76.0(49.7)$ & $95.7(20.7)$ & $<0.0001$ \\
\hline RR (breaths/min) & $8.0(11.0)$ & $18.0(6.8)$ & $<0.0001$ \\
\hline $\mathrm{O}_{2}$ saturation (\%) & $86.1(30.2)$ & $98.1(3.8)$ & $<0.0001$ \\
\hline GCS & $5.8(4.6)$ & $13.6(3.4)$ & $<0.0001$ \\
\hline
\end{tabular}

the ISS is an anatomically based scoring system based on injuries to major body regions including the head and neck, face, chest, abdomen and pelvic contents, extremities and pelvic girdle and external body surface. ${ }^{910}$ The ISS is useful for assessment following motor vehicle collisions. The ISS algorithm incorporates points from each region with a maximum score of 75 (higher values represent more serious trauma). Finally, the RTS can be used by prehospital emergency personnel to assist with the triage of injured patients. The variables include RR, SBP and GCS ${ }^{11}$ and the unweighted RTS is calculated by adding the values assigned for each parameter against a maximum possible score of 12 .

\section{Data analysis}

Continuous variables are described using mean and SD. Similarly, categorical variables are described using frequency and percentage. Parametric tests were used for the comparisons between groups: $\chi^{2}$ test in the case of categorical variables and the test in the case of continuous variables. Correlations were tested using Spearman test. Sensitivity and specificity were plotted using the receiver operating characteristic curve. The discriminate power of REMS, RTS, ISS and SI was compared using the AUC. Statistically significant associations and differences were identified by $\mathrm{p}$ values of less than 0.05 . All analyses were conducted using SAS V.9.3 (copyright 2002-2008 by SAS Institute Inc, Cary, North Carolina, USA).

\section{RESULTS}

Of the patients studied, 3489 (94.8\%) lived and 191 $(5.2 \%)$ died. Characteristics of the study sample are included in table 2. Patients who lived had a mean age of $36.5 \pm 17.0$ years, $73.7 \%$ were men, $62.5 \%$ were Caucasian, the average REMS was $3.4( \pm 3.2)$ and the average length of stay was $7.6( \pm 15.4)$ days. Patients who died had a mean age of 43.7 years, $77 \%$ were men,
59.2\% were Caucasian, the average REMS was 11.8 and the average length of stay was 4.4 days.

The study team looked at the distribution of patient mortality by each incremental REMS score (1-26). Based on this distribution, the authors used natural cut-offs as well as their clinical judgement to develop the REMS groupings used in the study. A higher REMS was associated with increased mortality $(\mathrm{p}<0.0001$, table 3$)$. Patients with REMS less than 6, from 6 to 9 and greater than 9 had mortalities of $0.9 \%, 6.7 \%$ and $39.3 \%$, respectively. An increase of 1 point within the 26-point REMS was associated with an OR of 1.51 for in-hospital death (95\% CI 1.45 to 1.58$)$.

Patients with low REMS scores who died presented to the ED with lower median GCS than those who survived. The median GCS for patients with REMS of 3-5 and 6-9 who died was $1.5(\mathrm{p}<0.0001)$ and $8(\mathrm{p}<0.0001)$ points lower than those who lived. Patients with high REMS scores who lived presented to the ED with a higher median age than those who died. The median age for patients with REMS of 16-19 and 20-21 who lived was

Table 3 REMS score characteristics $(p<0.0001)$ for 3680 patients with trauma

\begin{tabular}{lccc}
\hline REMS & Alive (N) & Dead (N) & Mortality (\%) \\
\hline $0-2$ & 1749 & 6 & 0.3 \\
$3-5$ & 999 & 20 & 2 \\
$6-9$ & 547 & 39 & 6.7 \\
$10-11$ & 110 & 28 & 20.3 \\
$12-13$ & 53 & 26 & 32.9 \\
$14-15$ & 22 & 18 & 45 \\
$16-19$ & 8 & 33 & 80.5 \\
$20-21$ & 1 & 13 & 92.9 \\
$22-26$ & 0 & 8 & 100 \\
Total & 3489 & 191 & 5.2 \\
\hline REMS, Rapid Emergency Medicine Score.
\end{tabular}


Table 4 Injury scores for 3680 patients with trauma

\begin{tabular}{lllll}
\hline & N & $\begin{array}{l}\text { Dead mean } \\
\text { (SD) }\end{array}$ & $\begin{array}{l}\text { Alive mean } \\
\text { (SD) }\end{array}$ & p Value \\
\hline REMS & 3680 & $11.8(5.4)$ & $3.4(3.2)$ & $<0.0001$ \\
SI & 3633 & $0.79(0.40)$ & $0.69(0.22)$ & $<0.0001$ \\
RTS & 3680 & $3.5(2.7)$ & $7.4(1.3)$ & $<0.0001$ \\
ISS & 3671 & $30.0(14.6)$ & $11.3(10.0)$ & $<0.0001$ \\
\hline
\end{tabular}

ISS, Injury Severity Score; REMS, Rapid Emergency Medicine Score; RTS, Revised Trauma Score.

$45.5(\mathrm{p}=0.01)$ and $50(\mathrm{p}=0.13)$ years older than those who died.

Secondarily, REMS was compared to three additional injury severity scoring systems (table 4). REMS (AUC $0.91 \pm 0.02$ ) was found to be similar to RTS (AUC 0.89

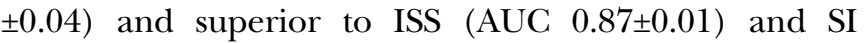
(AUC $0.55 \pm 0.31$ ) in predicting in-hospital mortality (figure 1). The mean SI for patients who lived was 0.69 $( \pm 0.22)$ and $0.79( \pm 0.40)$ for those who died. The mean RTS for patients who lived was $7.4( \pm 1.3)$ and $3.5( \pm 2.7)$ for those who died. Finally, the mean ISS score for patients who lived was $11.3( \pm 10.0)$ and $30.0( \pm 14.6)$ for those who died.

While multivariable logistic regression indicated that heart rate and RR individually did not predict mortality, the other four REMS parameters did (table 5). GCS was the strongest predictor of mortality (OR $0.743,95 \%$ CI 0.71 to 0.78$)$. While not directly assessed by REMS, injury type also impacts patient mortality rates (table 6). Patients with penetrating trauma experienced higher mortality rates than those with blunt force trauma $(8.0 \%$ vs $4.4 \%, \mathrm{p}<0.0001)$.

\section{DISCUSSION}

Despite recent improvements in trauma systems and their resulting decrease in preventable deaths, trauma continues to be the leading cause of mortality for those under the age of 44 years in the USA. ${ }^{12}$ Trauma systems rely on imperfect and subjective tools to triage critically
Table 5 Multiple logistic regression for all parameters in REMS

\begin{tabular}{lllr}
\hline Variable & OR & 95\% Cl & p Value \\
\hline GCS & 0.743 & 0.711 to 0.777 & $<0.0001$ \\
$\mathrm{O}_{2}$ saturation (\%) & 0.961 & 0.940 to 0.982 & 0.0004 \\
Age (years) & 1.034 & 1.024 to 1.044 & $<0.0001$ \\
MAP (mm Hg) & 0.979 & 0.973 to 0.986 & $<0.0001$ \\
Heart rate (bpm) & 0.996 & 0.990 to 1.002 & 0.2179 \\
RR (breaths/min) & 1.001 & 0.978 to 1.025 & 0.9023 \\
\hline
\end{tabular}

GCS, Glasgow Coma Scale; MAP, mean arterial pressure; RR respiratory rate.

injured patients to the appropriate centre. Currently, emergency medical service (EMS) and trauma care providers utilise a variety of factors such as blood pressure, RR, GCS, as well as mechanism of injury to prioritise responses and resources. With increasing rates of ED and trauma diversion, particularly in urban settings, efficient allocation of resources is more crucial than ever. ${ }^{13}$ Organised and inclusive trauma systems depend on patients being routed to the closest and most appropriate centre capable of caring for the patient. Any tool that can match true severity with the highest possible resource within the system may ensure that higher level centres are able to concentrate on patients most in need of their capabilities. In particular, REMS appears to provide an effective balance between the predictive ability and the practical application which are necessary for the trauma setting. Unlike systems used elsewhere in the hospital, REMS does not require invasive or timeconsuming lab values such as lactate, base deficit, mixed venous oxygen saturation or cutaneous tissue oxygen saturation. ${ }^{14-19}$ Rather, REMS utilises readily available parameters available both in the prehospital environment and in the ED.

The current body of literature related to REMS examines its application among non-surgical medicine patients. Studies conducted by Olsson et al have concluded that use of the REMS among non-surgical patients in the ED is powerful in predicting both

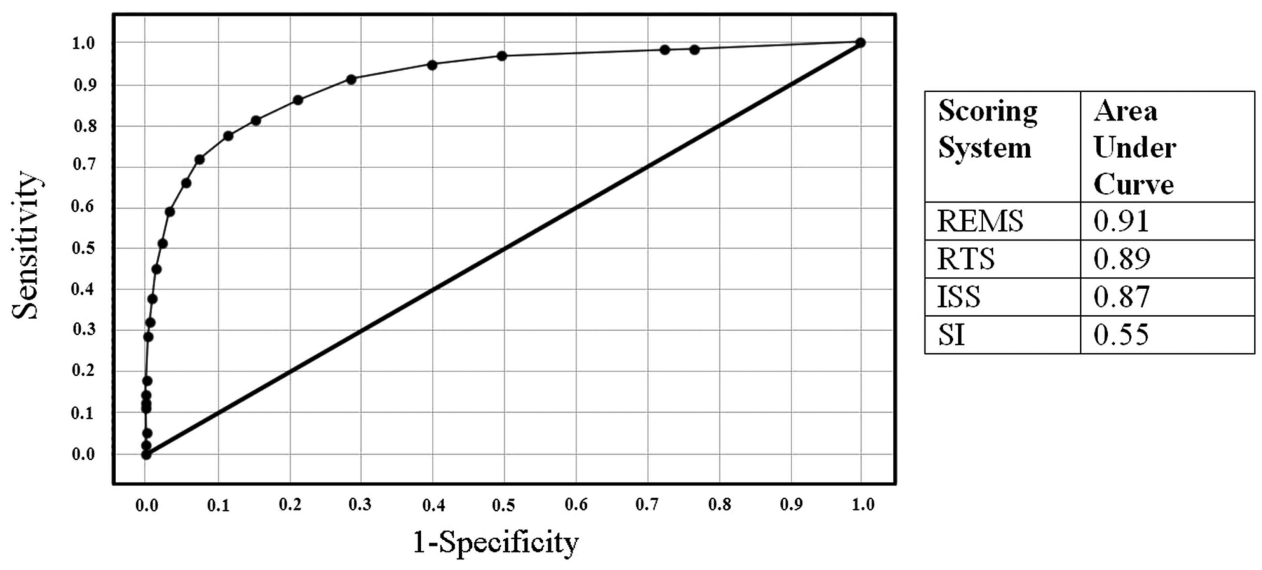

Figure 1 Rapid Emergency Medicine Score (REMS) receiver operating characteristic curve (area under the curve=0.91). 


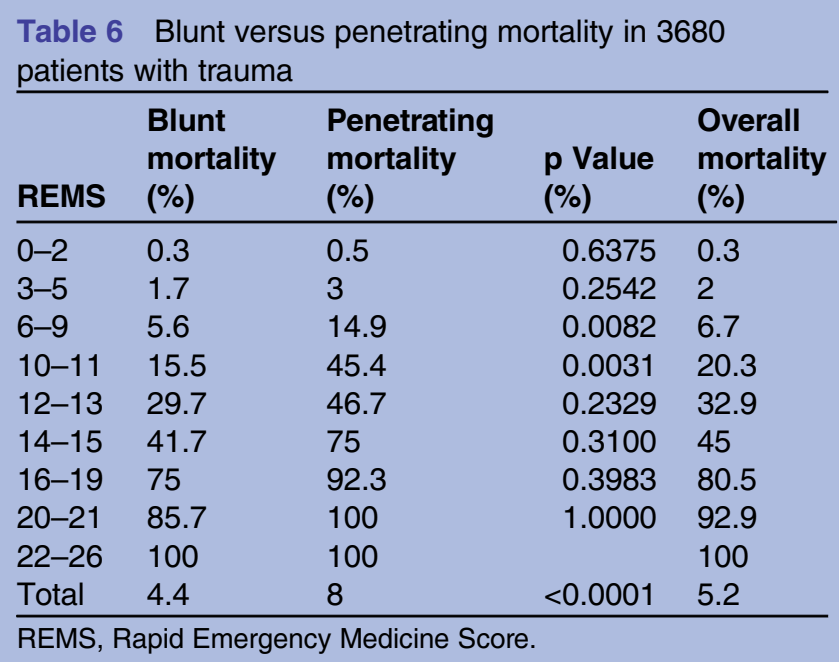

in-hospital and long-term mortality. In their studies, their research showed that all six REMS parameters were predictive of mortality. Similarly, Goodcare et al found REMS to be effective in predicting mortality among medicine patients, although, age, GCS and oxygen saturation were the only REMS parameters that strongly correlated with mortality. Our study indicates that applying REMS to the trauma population yields similar results and that REMS is a strong predictor of mortality.

It is important to note that REMS, like other trauma scoring methods, was originally devised using population-level data. Applying REMS at the individual patient level yields an estimated mortality percentage; because actual outcomes are binary (ie, alive or dead), it would not be unexpected for a limited number of patients with low REMS to die and those with high REMS to live.

In this study, REMS performed similar to or better than all other measures in predicting mortality. ISS was nearly as predictive of mortality but is a retrospective system whose score can only be determined after diagnosis. ISS is therefore better suited as a benchmark for comparison (between patient groups or trauma centres) and not practically useful as a triage tool. While SI, which is calculated using two readily available vitals (heart rate and SBP), is both simple and fast to utilise, it severely underperformed REMS in predicting mortality. Unweighted RTS was found to be the most effective alternative to REMS as a triage tool; its strength in predicting mortality was similar to that of REMS, but RTS uses only a subset of REMS measures (GCS, SBP and RR) and a 0-4-point scale for each variable. When compared with REMS, RTS substitutes a small amount of predictive ability in return for simplicity.

This study expands the usefulness of REMS in the ED, demonstrating its capability for predicting mortality in the trauma population. However, since REMS components and values were derived using data from a medicine population, further analysis of the applicability to a trauma population may be beneficial. Underlying reasons may include that medicine patients are more likely to have chronic comorbidities that may contribute to differing baseline vitals leading to a score variation. In addition, medicine patients may have a greater portion of their score attributable to age in comparison to that of the trauma population, which often consists of younger, otherwise healthy adults. Similar to Goodcare et al, this study found that the REMS components correlated with mortality were GCS, oxygen saturation, age and MAP; of those, GCS was found to have the strongest predictor of mortality among the trauma population, while heart rate and RR did not exhibit any statistical significance.

Analysis suggests that GCS may be underweighted in the REMS calculation for patients with trauma. At the lower end of the REMS scale (scores of 3-5 and 6-9), patients who died presented with a lower average GCS than those who lived. For REMS of 3-5, the average GCS for those who died was 11.1 (9-12 is classified as a moderate head injury $\left.{ }^{20}\right)$ compared to $14.2(13-15$ is classified as a mild head injury) for those who lived. For REMS of 6-9, the average GCS for those who died was 8.4 ( $\leq 8$ is classified as a severe head injury) compared to 11.5 (moderate head injury) for those who lived. In a study of geriatric trauma, Champion $e t a l^{21}$ found a difference in mortality between patients with trauma older than $65(19 \%)$ and those younger than $65(9.8 \%)$. This difference in mortality may be due to the factor of age and the weight it carries within the REMS calculation methodology. However, our findings suggest that age may be overweighted in the REMS calculation for patients with trauma. At the higher end of the REMS scale (scores of 16-19 and 20-21), patients who survived presented with a greater average age; in these cases, a greater portion of the composite REMS score came from age.

REMS was derived from the medicine population; as a result, the scoring system does not differentiate between injury types. For the trauma population, however, injury type is a significant factor in mortality prediction. A significant difference in average mortality rates was demonstrated in patients presenting with blunt $(4.4 \%)$ versus penetrating $(8 \%)$ injury types. Differences in mortality rates by injury type persist at all levels of REMS.

This study, which analysed a large number of patients with trauma within a single hospital system, does possess several limitations. First, this was a retrospective analysis. As with any such study, potential disadvantages include the possibility of selection bias. Second, the study excluded 158 potential records from the analysis due to missing patient data required to calculate REMS. Forty-seven patients were excluded from the calculation due to missing non-REMS injury scores (ie, RTS, ISS, SI). Finally, because the study is based on data from a single urban academic ACS level 1 trauma centre, attempts to extrapolate results to other trauma populations may not be reliable. Given the focused population, opportunities exist for future expansion and improvement, such as 
conducting a prospective study on the predictive capability of REMS on mortality, and using a larger, more diverse trauma dataset. Evaluating REMS in the prehospital setting and monitoring the change in REMS from initial EMS contact to trauma centre arrival may prove to be useful for EMS and for the ED by enhancing the effectiveness of the triage process, the appropriate routing of patients and the utilisation of trauma resources.

\section{CONCLUSIONS}

Though initially designed for the medicine population, REMS was found to be a strong predictor of in-hospital mortality for the trauma population. REMS performed similarly to RTS and outperformed several other traditionally used trauma scales including ISS and SI. This study also indicates potential opportunities to better apply REMS to the trauma population. REMS components heart rate and RR were found to have no statistically significant contribution in mortality prediction, while age and GCS were overweighted or underweighted in the REMS calculation, respectively. Injury type is an important predictor of mortality in patients with trauma, and it was not included in the REMS calculation. Each of these represents an opportunity for future study.

Acknowledgements The authors would like to thank Alvina Fant and Tracy McDonald for their assistance obtaining and processing the trauma registry data, as well as the University of Kansas Hospital Trauma Department for their ongoing support.

Contributors BFI participated in designing and coordinating the execution of the study, conducted the chart review and drafting of the manuscript. NJT participated in designing and coordinating the execution of the study and drafting of the manuscript. MAH participated in coordinating the execution of the study. NN performed the statistical analysis. MM participated in the design of the study and drafting of the manuscript. CMC participated in the design of the study and drafting of the manuscript. All authors read and approved the manuscript.

Funding This research received no specific grant from any funding agency in the public, commercial or not-for-profit sectors.

Competing interests None.

Ethics approval University of Kansas Medical Center Human Subjects Committee.

Provenance and peer review Not commissioned; externally peer reviewed.

Data sharing statement No additional data are available.

Open Access This is an Open Access article distributed in accordance with the Creative Commons Attribution Non Commercial (CC BY-NC 3.0) license, which permits others to distribute, remix, adapt, build upon this work non-commercially, and license their derivative works on different terms, provided the original work is properly cited and the use is non-commercial. See: http://creativecommons.org/licenses/by-nc/3.0/

\section{REFERENCES}

1. Centers for Disease Control (CDC). Ten leading causes of death and injury (charts). 2006; 2010. http://www.cdc.gov/injury/wisqars/ LeadingCauses.html

2. Olsson T, Terent A, Lind L. Rapid Emergency Medicine Score can predict long-term mortality in non-surgical emergency department patients. Acad Emerg Med 2004;11:1008-13.

3. Knaus WA, Draper EA, Wagner DP, et al. APACHE II: a severity of disease classification system. Crit Care Med 1985;13:818-29.

4. Olsson T, Lind L. Comparison of the rapid emergency medicine score and APACHE II in non-surgical emergency department patients. Acad Emerg Med 2003;10:1040-8.

5. Olsson T, Terent A, Lind L. Rapid Emergency Medicine Score: a new prognostic tool for in-hospital mortality in non-surgical emergency department patients. J Intern Med 2004;255:579-87.

6. Goodcare S, Turner J, Nicholl J. Prediction of mortality among emergency medical admissions. J Emerg Med 2006;23:372-5.

7. Birkhahn $\mathrm{RH}$, Gaeta TJ, Terry $\mathrm{D}$, et al. Shock index in diagnosing early acute hypovolemia. Am J Emerg Med 2005;23:323-6.

8. Cannon CM, Braxton CC, Kling-Smith M, et al. Utility of the shock index in predicting mortality in traumatically injured patients. J Trauma 2009;67:1426-30.

9. Goris RJ. The injury severity score. World J Surg 1983;7:12-8.

10. Baker SP, O'Neill B, Haddon W Jr, et al. The injury severity score: a method for describing patients with multiple injuries and evaluating emergency care. J Trauma 1974;14:187-96.

11. Champion HR, Sacco WJ, Copes WS, et al. A revision of the trauma score. J Trauma 1989;29:623-9.

12. National Institute of General Medical Sciences. Fact sheet: trauma, shock, burn, and injury-facts and figures. NIGMS.NIH.gov/ Publications/trauma_burn_facts.htm (accessed 15 Feb 2008).

13. Begley CE, Chang $\bar{Y}$, Wood RC, et al. Emergency department diversion and trauma mortality: evidence from Houston, Texas. J Trauma 2004;57:1260-5.

14. Blow O, Magliore L, Claridge JA, et al. The golden hour and the silver day: deterioration and correction of occult hypoperfusion within 24 hours improves outcome from major trauma. $J$ Trauma 1999;47:964-9.

15. Claridge JA, Crabtree TD, Pelletier SJ, et al. Persistent occult hypoperfusion is associated with a significant increase in infection rate and mortality in major trauma patients. J Trauma 2000;48:8-14.

16. Andel D, Kamolz LP, Roka J, et al. Base deficit and lactate: early predictors of morbidity and mortality in patients with burns. Burns 2007;33:973-8.

17. Kazorian KK, Del Guericio LR. The use of mixed venous blood gas determinations in traumatic shock. Ann Emerg Med 1980;9:179.

18. Bakker J, Gris T, Coffernils M, et al. Serial blood lactate levels can predict the development of multiple organ failure following septic shock. Am J Surg 1996;171:221-6.

19. Cohn SM, Nathens AB, Moore FA, et al. StO2 in Trauma Patients Trial Investigators. Tissue oxygen saturation predicts the development of organ dysfunction during traumatic shock resuscitation. J Trauma 2007;62:44-54.

20. Centers for Disease Control (CDC). Glascow Coma Scale. 2013 http://www.bt.cdc.gov/masscasualties/gscale.asp

21. Champion HR, Copes WS, Buyer D, et al. Major trauma in geriatric patients. Am J Public Health 1989;79:1278-82. 